\title{
TRABAJO DOCENTE, ESCOLARIZACIÓN Y DESIGUALDADES SOCIALES: CONTRIBUCIONES Y DEBATES EN LA INVESTIGACIÓN EDUCATIVA EN ARGENTINA
}

\author{
Alejandro Vassiliades \\ Universidad de Buenos Aires(UBA) y Universidad Nacional de la Plata (UNLP), Argentina
}

RESUMEN: Este artículo se propone realizar un análisis crítico y sistemático de la producción científica acerca de los vínculos entre trabajo docente, escolarización y desigualdades sociales en el período 1985-2015 en Argentina, con el objeto de examinar los principales debates conceptuales y teóricometodológicos, como así también sus contribuciones para las discusiones actuales en el campo de la educación. Para ello, este trabajo reconstruye tres núcleos de debate: las contribuciones de la reformulación de los abordajes estructurales y del redireccionamiento de la mirada hacia los sujetos y las instituciones escolares; las discusiones surgidas de la incorporación de preocupaciones centradas en la heterogeneidad y el dinamismo de las identidades sociales; y los debates respecto del lugar de los contextos de pobreza en las instancias de formación y trabajo docente, ligados nuevas aproximaciones a los vínculos entre docencia, escolarización y desigualdad. Palabras clave: Trabajo docente. Desigualdad social. Investigación educativa. Procesos de escolarización. Campo educativo.

\section{TRABALHO DOCENTE, ESCOLARIZAC̣ÃO E DESIGUALDADES SOCIAIS: CONTRIBUIC̣̃̃ES E DEBATES DA PESQUISA EDUCACIONAL NA ARGENTINA}

RESUMO: Este artigo propõe-se a realizar uma análise crítica e sistemática da produção científica acerca dos vínculos entre trabalho docente, escolarização e desigualdades sociais no período entre 1985 e 2015, na Argentina, com o objetivo de examinar os principais debates conceituais e teórico-metodológicos, bem como suas contribuições para as discussões atuais no campo da educação. Para isso, este trabalho reconstrói três núcleos de debate: as contribuições da reformulação das abordagens estruturais e

\footnotetext{
"Doctor en Educación por la Universidad de Buenos Aires (UBA), Argentina. Investigador Asistente del Consejo Nacional de Investigaciones Científicas y Técnicas (CONICET), Argentina, con sede en el Instituto de Investigaciones en Humanidades y Ciencias Sociales (CONICET-UNLP). Docente e investigador de la Universidad de Buenos Aires y de la Universidad Nacional de La Plata. E-mail: < alevassiliades@gmail.com > .
} 
do redirecionamento do olhar para os sujeitos e as instituições escolares; as discussões surgidas a partir da incorporação de preocupações centradas na heterogeneidade e no dinamismo das identidades sociais; e os debates a respeito do lugar dos contextos de pobreza nas instâncias de formação e trabalho docente, ligados a novas aproximações dos vínculos entre docência, escolarização e desigualdade.

Palavras-chave: Trabalho docente. Desigualdade social. Pesquisa educativa. Processos de escolarização. Campo educativo.

\section{TEACHING WORK, SCHOOLING AND SOCIAL INEQUALITIES: CONTRIBUTIONS AND DEBATES IN THE EDUCATIONAL RESEARCH IN ARGENTINA}

ABTRACT: This article intends to present a critical and systematic analysis of the scientific production on the links between teaching work, schooling and social inequalities between 1985 and 2015 in Argentina, with the aim of examining the main conceptual and theoretical-methodological debates, as well as their contributions for the current discussions in the educational field. This paper will approach three cores of debate: the contributions of the reformulation of structural approaches and the reframing of the looking to the subjects and schools; the discussions from the incorporation of the questions centred in the heterogeneity and dynamism of social identities; and the debates centred on the place of contexts in the instances of teachers' training and work, linked to new approaches to the links between teaching, schooling and inequality.

Keywords: Teaching work. Social inequality. Educational research. Schooling processes. Educational field.

\section{INTRODUCCIÓN}

La cuestión de los múltiples vínculos entre el trabajo docente, los procesos de escolarización y las desigualdades sociales ha constituido, posiblemente, una de las mayores preocupaciones del campo de la investigación educativa en Argentina en las últimas décadas. El modo en que se relacionan y se configuran mutuamente la tarea de enseñar y el problema de la desigualdad ha integrado las agendas de las diferentes tradiciones y áreas de conocimiento con las que la pedagogía ha establecido articulaciones.

En el marco de la construcción del estado del arte de un proyecto de investigación en curso ${ }^{1}$, este artículo se propone realizar un análisis crítico y sistemático de la producción científica acerca de los vínculos entre trabajo docente, escolarización y desigualdad en el período 1985-2015 con el objeto de examinar los principales debates 
conceptuales y teórico-metodológicos en la investigación, como así también sus principales contribuciones para las discusiones actuales en el campo de la educación.

Este artículo intentará dar cuenta de cómo las producciones académicas relevadas en el período 1985-2015 en Argentina han ido inscribiéndose en los procesos de conformación del campo pedagógico como campo de saber y de poder, y de las reconceptualizaciones y reformulaciones de los lenguajes teórico-metodológicos de la investigación en educación (HILLERT, 2009; LLOMOVATTE, 2009; SUÁREZ, 2008) ${ }^{2}$. Para ello, se han relevado y sistematizado un corpus de 35 investigaciones y estudios desarrollados en ese período para realizar una revisión crítica y sistemática de la producción científica y académica acerca de los vínculos entre el trabajo docente, los procesos de escolarización y la desigualdad, sobre la base de una serie de coordenadas:

- Los marcos conceptuales que las investigaciones y estudios utilizan para aproximarse a los vínculos entre escolarización, trabajo docente y desigualdad.

- Las definiciones teórico-metodológicas tomadas por las investigaciones y estudios relevados para producir conocimiento acerca de los vínculos entre escolarización, trabajo docente y desigualdad.

- Las contribuciones y nuevos interrogantes aportados por las investigaciones y estudios relevados en relación a los vínculos entre escolarización, trabajo docente y desigualdad, como así también los debates colocados en el campo de la educación por parte de esas producciones científicas.

- Los sentidos acerca de la escolarización, el trabajo docente y la desigualdad que las investigaciones y estudios relevados construyen. Estos significados se inscriben en los debates que surcan el campo pedagógico como campo dinámico de saber y de poder.

A partir de estos ejes y de un trabajo interpretativo de análisis de contenido de los textos relavados, el artículo reconstruye tres núcleos de discusión en la producción académica y científica en Argentina sobre los vínculos entre el trabajo docente, los procesos de escolarización y la desigualdad. En primer lugar, el texto recorrerálos debates y contribuciones de la reformulación de los abordajes estructurales y del redireccionamiento de la mirada hacia los sujetos 
y las instituciones escolares. En segunda instancia, el trabajo analizará las discusiones surgidas de la crítica a las miradas generalizantes sobre el trabajo docente, abordando los modos en queel campo de la investigación educativa incorporó preocupaciones centradas en la heterogeneidad y el dinamismo de las identidades sociales a la hora de analizar los procesos de escolarización de sujetos en contextos de desigualdad. Finalmente, en tercer término, el artículo recorrerá los modos en que la producción académica situó el debate respecto del lugar de los contextos de pobreza en las instancias de formación y trabajo docente, reconstruyendo los modos que se plantearon nuevas aproximaciones al análisis de los vínculos entre docencia, escolarización y desigualdad.

\section{ENTRE LA ESTRUCTURA SOCIAL Y LOS SUJETOS: DEBATES ACERCA DE LAS DINÁMICAS DE LA DESIGUALDAD EN LA INVESTIGACIÓN EDUCATIVA EN ARGENTINA}

En Argentina, las investigaciones educativas orientadas a indagar acerca de los procesos de construcción de la desigualdad tienen uno de sus hitos importantes en el trabajo pionero de Braslavsky (1985) en torno de las dinámicas discriminadoras y diferenciadoras del sistema educativo. Este estudio supuso un valioso aporte que permitió profundizar la ruptura con el optimismo pedagógico que habían inaugurado algunos desarrollos del campo de la sociología de la educación, a partir de analizar el modo en que el sistema educativo argentino desarrolló formas de integración escolar diferenciadas que perpetuaban las desigualdades sociales a través de circuitos o segmentos de escolarización para los distintos sectores sociales. Asimismo, señaló la necesidad de interrogarse qué sucedía en el interior de las escuelas para abordar la cuestión de la desigualdad, abriendo interrogantes en torno de la distribución de las oportunidades entre diferentes escuelas, entre modalidades de enseñanza y entre las propias expectativas de las familias, trazando una serie de problemas que serían recuperados por investigaciones posteriores.

Este estudio se situó en continuidad con otros que, en el campo de la sociología de la educación, se ocuparon de estudiar los vínculos entre los procesos de escolarización y la desigualdad social. Esta problemática fue incorporándose de manera creciente en la agenda de la investigación socioeducativa internacional, registrando una importante presencia hacia el último tercio del siglo XX. En efecto, hacia fines de la década del '60 y principios de la década del '70 comenzaron a producirse una serie de trabajos que romperían 
con el optimismo pedagógico imperante hasta ese momento, proporcionando un análisis de las relaciones entre desigualdades sociales y los procesos de escolarización (BOWLES y GINTIS, 1976; BAUDELOT y ESTABLET, 1976). Producidos en diversos países y contextos, estos estudios rompieron con la idea de que las instituciones educativas eran lugares neutrales necesariamente dirigidos a la construcción de sociedades más igualitarias.

LainvestigacióndesarrolladaporBraslavsky(1985)mostrócómo, hacia mediados de la década de 1980, la segregación socioeconómica del sistema educativo argentino constituía una práctica habitual y generaba circuitos educativos diferenciados entre escuelas de diferente calidad en relación con el origen social de los alumnos. Así, la escuela cumplía un importante papel en la reproducción de las desigualdades sociales. Los circuitos diferenciados expresaban los modos en que la educación como derecho se encontraba desigualmente distribuida en los diferentes circuitos educativos, estableciendo articulaciones entre escolarización y origen socioeconómico de los alumnos, quienes -de acuerdo al sector social al que pertenecían- transitaban por circuitos paralelos sin contactos entre sí. La segmentación y la desarticulación de los sistemas educativos, principales categorías teóricas de la investigación desarrollada, constituían expresiones y modalidades específicas de sus dinámicas de diferenciación horizontal y vertical. La diferenciación horizontal se manifestaba en aquellas situaciones en las que establecimientos educativos que de acuerdo a la legislación debían ser iguales, poseían un currículum y ofrecen condiciones para el aprendizaje muy diferentes. La diferenciación vertical, por su parte, daba cuenta de la existencia de diversos órganos de orientación y conducción de cada nivel del sistema educativo operando en forma independiente, pudiendo configurar un sistema desarticulado. En consecuencia, la segmentación y desarticulación resultaban funcionales a la conservación del monopolio de la educación en grupos sociales minoritarios (BRASLAVSKY, 1985). Para la perspectiva teóricometodológica en la que se fundó esta investigación, el sentido de la desigualdad se condensaba en las dinámicas diferenciadoras de acceso al conocimiento escolar.

El estudio se apartó de los modelos reproductivistas clásicos al incorporar interpretativamente las perspectivas y representaciones de los sujetos sociales. Analizó una serie de efectos de algunos rasgos del trabajo docente -la antigüedad en el cargo, la estabilidad y la formación- en los procesos educativos, y el rendimiento escolar de los alumnos. También abordó el modo en que el modelo pedagógico 
de las instituciones priorizaba la transmisión de conocimientos o la socialización. El trabajo implicó un valioso aporte en términos de desmontar la construcción de la apariencia igualitaria del sistema escolar en Argentina (BRASLAVSKY, 1985).

Los estudios de sociología de la educación de los años siguientes pusieron el foco en la desigualdad como producción social y escolar, evidenciando cómo las instituciones educativas desarrollaban procesos que entraban en tensión con sus promesas de liberación e igualdad. De forma progresiva, el campo de la investigación educativa fue incorporando otras dimensiones de abordaje, como el análisis de la interacción maestro/alumno y el papel de la subjetividad de los actores sociales en la producción y reproducción de la desigualdad en los ámbitos educativos. La mirada se orientó hacia el interior de la "caja negra" de la escuela, dando cuenta de la revisión que el propio campo de la investigación educativa había hecho de los modelos objetivistas de tipo funcionalista o marxista que privilegiaban el punto de vista de la totalidad, incorporando corrientes teóricas de origen anglosajón como el interaccionismo simbólico, la etnometodología y la fenomenología social. En Argentina, el desplazamiento hacia los procesos, la subjetividad, las prácticas y experiencias de los sujetos sociales también tuvo lugar, en el campo educativo, con la incorporación de perspectivas que abrevaban en el campo de la antropología social.

Este énfasis en las perspectivas de los sujetos tuvo diversas expresiones en el campo de la investigación educativa. En el contexto de una creciente atención a las interacciones entre docentes y alumnos, algunos estudios indagaron acerca de las expectativas de los docentes respecto de sus alumnos y el modo en que ellas influyen en los procesos de escolarización, poniendo en relación los procesos de estigmatización social y el llamado "fracaso escolar", como así también otras dinámicas de exclusión (KAPLAN, 1992,1997). Las bajas expectativas parecían concentrarse en niños y niñas de sectores populares o subalternos en términos de etnia o género (MORGADE, 1992, 2001, 2006). Los aportes para estudiar la desigualdad como producción social se centraron en el estudio de la naturalización de las diferencias y categorías con las que el mundo es aprehendido, y en cómo su producción y reproducción se basaba en el desconocimiento de su carácter arbitrario (TENTI FANFANI, 2007).

Apartándose de las perspectivas deterministas y biologicistas sobre el éxito y el fracaso escolar propias de las décadas del ' 50 y '60, las investigaciones socioeducativas producidas en los últimos veinte años mostraron que las representaciones de los profesores 
operan como veredictos sobre los límites de los alumnos con relación al éxito o fracaso escolar, estructurando un efecto destino (KAPLAN, 2008). Estas contribuciones se sustentaron en la idea de que las desigualdades en sociedades capitalistas no radicaban en la genética sino en la desigual distribución de las condiciones materiales y simbólicas que caracteriza a las sociedades y las escuelas. Estos trabajos apuntaron que las representaciones subjetivas de los profesores debían ser entendidas en una trama de configuraciones sociales presentes y pasadas, y que el talento y la inteligencia debían ser des-sustancializados y desnaturalizados como parámetros entre los exitosos y los fracasados escolares, por tratarse de una construcción social y escolar. En este sentido, los juicios de los docentes respecto de sus estudiantes transforman las diferencias sociales entre ellos en desigualdades vividas como naturales (KAPLAN, 2008).

Asimismo, un conjunto de investigaciones se aproximó a los modos en que la escuela se entrama con la producción y la reproducción de la desigualdad analizando cómo los sujetos y las instituciones escolares dan cuenta de un escenario de fragmentación social. Estos estudios parten de la redefinición del campo de las ciencias sociales dado el escenario de reestructuración de las relaciones y marcos regulatorios de la acción de individuos e instituciones hacia fines del siglo XX. En el caso argentino, estas revisiones también se afirmaron en la idea de que las expresiones escolares de la desigualdad ya no podrían explicarse sólo en términos de diferenciaciones de orden socioeconómico, sino que la diversidad de posicionamientos y estrategias de los sujetos escolares las excedían. Estas nuevas dinámicas fueron acompañadas de una presunta crisis del Estado como figura clave en la construcción de un espacio de sentido para el conjunto de los individuos y de las instituciones. La escuela, asociada a este espacio común, también entró en crisis como institución portadora de una propuesta universalista y como dispositivo de regulación social.

Desde estas coordenadas, la investigación desarrollada por Tiramonti (2004) planteó la existencia de un campo educativo fragmentado para el nivel medio de enseñanza en el que los actores se mueven dentro de un espacio relativamente cerrado que marca los límites de sus opciones. La categoría fragmentación educativa, como lente a través de la cual observar la cuestión de la desigualdad, se sustentó metodológicamente en el análisis del papel que desempeñan las expectativas familiares, los recursos institucionales, los capitales culturales de las familias y las estrategias de los diferentes sectores sociales ante la situación en la que deben desenvolverse. Asimismo, 
mostró la insuficiencia de una mirada anclada al concepto de clase para indicar las líneas de quiebre entre uno y otro fragmento, si bien las nuevas desigualdades se montan sobre las anteriores.

El fragmento es un espacio autorreferido en el interior del cual se pueden distinguir continuidades que lo delimitan y diferencias que dan cuenta de la heterogeneidad de estos espacios, con escasa o nula articulación entre ellos. Se trata de una frontera de referencia para las opciones escolares de aquellos que lo integran (TIRAMONTI, 2004). Algunos trabajos en el marco de dicha investigación analizan los factores que fragmenta el cuerpo docente, la construcción de sus perfiles y las estrategias que reúnen escuelas con docentes (POLIAK, 2004), y las representaciones docentes sobre lo público y los valores asociados a esta esfera (ARROYO, 2004). Estas investigaciones comparten la advertencia de que una mayor autonomía o una menor regulación estatal abrió paso a situaciones de creciente heterogeneidad institucional respecto de la educación que las escuelas ofrecen, configurando perfiles que interactúan con otras formas de desigualdad de forma compleja. La principal premisa teórico-metodológica que organizó estos estudios fue el abandono de las miradas estructuralistas que establecían continuidades entre los posicionamientos sociales y las desigualdades educativas, avanzando en la consideración de los sujetos y del impacto que tienen las nuevas condiciones de existencia sobre las subjetividades y respecto de los sentidos que se construyen sobre la desigualdad.

\section{HOMOGENEIDADES EN DEBATE: LA DESIGUALDAD COMO RELACIÓN EN EL CAMPO DE LA INVESTIGACIÓN EDUCATIVA EN ARGENTINA}

Un conjunto de producciones científicas y académicas en el campo de la investigación educativa en Argentina han puesto en debate las miradas generalizantes sobre la escolarización, el trabajo docente y las desigualdades, en tanto los sentidos que se asocian a estos significantes son múltiples. Así, comenzó a resultar conceptualmente inviable asumir una homogeneidad de posiciones en los sujetos escolares. Un número de investigaciones sobre la escolarización en contextos de pobreza han contribuido a la construcción de perspectivas que atiendan a las singularidades y complejidades de los vínculos entre docencia, procesos de escolarización y construcción de la desigualdad.

Una de los debates principales que, en este marco, se han planteado se refiere a la necesidad de desplazar una perspectiva sobre la desigualdad planteada en términos casi estrictamente económicos hacia una mirada de las desigualdades en términos más móviles y 
flexibles que las viejas divisiones de clase social o de posiciones de poder (DUSSEL, 2005). Así, un conjunto de estudios e investigaciones fueron asumiendo de forma creciente que las nociones de igualdad y desigualdad suponen una relación, descartando la posibilidad de considerarlas como esencias fijas e inmutables. La igualdad no sería entonces un estado estático o definido de una vez y para siempre, sino un conjunto de relaciones perdurables y fuertes que se establecen entre sujetos y que abarcan diferentes ámbitos, entre los que se encuentra el educativo. El carácter relacional de la desigualdad llevó a preguntarse por el conjunto de la sociedad y no solamente por la frontera que demarca a los incluidos de los excluidos, en tanto se trata de un problema político y social que está en el núcleo de las instituciones y las subjetividades (DUSSEL, 2005). En el marco de esta perspectiva relacional, algunas investigaciones se apartaron de la intención de determinar de modo excluyente quiénes están incluidos y quienes no, planteando que existe una condición fronteriza que da cuenta de los bordes, límites, pasajes, cruces y márgenes que habiliten miradas más complejas de los procesos de pauperización y su expresión en la vida cotidiana de familias e instituciones escolares (REDONDO y THISTED, 1999).

En este marco, un debate instalado por algunas producciones académicas en el campo de la investigación educativa en Argentina, es si las condiciones de pobreza y el desempeño de tareas asistenciales eran un obstáculo para la tarea de enseñar. Algunos trabajos han argumentado que estas nociones no pueden pensarse de modo dicotómico, como si enseñar y asistir fueran cuestiones opuestas (ANTELO, 2009). Las tareas llamadas "asistenciales" son muchas veces habilitantes y no obstaculizadoras de la enseñanza (REDONDO, 2004). Además, un número de estudios ha enfatizado que la calidad de la experiencia educativa que ciertas escuelas proponen no tiene necesariamente que ver con el origen social de los alumnos que asisten a ellas, cuestionando algunas hipótesis construidas por el campo de la investigación educativa años atrás. Desde una perspectiva interpretativa y etnográfica, estos trabajos han avanzado en abordar la construcción propiamente escolar y pedagógica de la desigualdad, mostrando cómo las instituciones educativas pueden habilitar otros horizontes o producir sus propias marcas exclusoras. En esta línea, investigaciones como la de Redondo y Thisted (1999) discuten la afirmación de que las escuelas "en los márgenes" están atrapadas en el "círculo vicioso de la pobreza", y que asocia esta situación y la falta de recursos pedagógicos a la baja calidad de los aprendizajes (TENTI FANFANI, 1993), planteando una relación determinista entre el empobrecimiento social y el empobrecimiento educativo. 
Desde el campo de la antropología social, y a partir de una investigación etnográfica, Padawer (2008) analiza una iniciativa docente que discute la organización por grados en la escuela primaria, apuntando que ella no sólo refiere a cuestiones técnico-didácticas sino también a una expresión contemporánea de un debate histórico sobre los principios políticos que estructuran el sistema de instrucción moderno en relación con la desigualdad social. Su investigación muestra da cuenta de la construcción de diversas posiciones en los maestros, mostrando cómo algunos sostienen la idea de que la pobreza producía un ambiente cultural "atrasado" en los hogares de los niños y que esa situación producía el fracaso escolar, mientras que otros asumen que dichas condiciones son un condicionante no definitivo y que hay diversos efectos del planteo de criterios de agrupamiento de los alumnos. La autora muestra que los maestros desarrollan, en tanto sujetos sociales, reflexiones complejas acerca de su papel en la discusión a la desigualdad social, discutiendo su reproducción en las prácticas que despliegan (PADAWER, 2008).

Del mismo modo, Redondo (2004) da cuenta de modos heterogéneas de asumir el trabajo de enseñar en docentes que trabajan con estudiantes que viven en condiciones de pobreza: quienes buscan compensar las carencias de sus alumnos a través del afecto y de la inculcación de buenos hábitos, quienes miran con indiferencia lo que les acontece y quienes reconocen la situación de desigualdad y la nombran desde la convicción de producir una reparación a través de la enseñanza, a partir del reconocimiento de la dignidad y los derechos. En algunos casos, la configuración de la identidad docente se funda en el vínculo con los alumnos nombrados como "carentes" y en cubrir aquello que les falta, sosteniendo en ocasiones responsabilidades que le competen al Estado.

De este modo, el campo de la investigación educativa avanzó en mostrar la heterogeneidad de situaciones y posiciones que se despliegan en los procesos de escolarización de sectores que viven en condiciones de pobreza (REDONDO y THISTED, 1999; REDONDO, 2004; TIRAMONTI, 2004; SOUTHWELL, 2008; VASSILIADES, 2012). En estos trabajos se da cuenta de los diversos modos en que los docentes pueden asumir perspectivas que plantean que los atributos que los alumnos traen son marcas indelebles de origen que predicen futuros frente a los cuales la escuela poco podría hacer, mientras que en otras instancias se construyen espacios para que el tránsito de los estudiantes por ella no esté fijado de antemano y habilite otros horizontes. Así, el abordaje de la docencia como posición 
(SOUTHWELL, 2009; VASSILIADES, 2012; SOUTHWELL y VASSILIADES, 2014) buscó indagar los modos múltiples y dinámicos en que los maestros y profesores asumen el trabajo de enseñar.

En este marco, en una investigación reciente se indagó el modo en que los directores caracterizan a sus alumnos y al perfil de sus instituciones (SOUTHWELL, 2008). Las escuelas indagadas registran la profundización de los factores de desigualdad social ocurrida en la década anterior, produciendo sus propias marcas exclusoras. Uno de los aspectos problemáticos referidos a estos procesos tiene que ver con que, en sus posicionamientos para definir lo que hacen o pueden hacer, los directivos asumen posturas variadas en torno de la homogeneidad o la heterogeneidad de sus estudiantes: en algunos casos, una de ellas es recuperada como valor; en otros, como obstáculo; en otros, son atribuidas al aislamiento o tamaño de la institución.

Las investigaciones recientes en el campo de la investigación socioeducativa han indagado acerca de las relaciones entre escuela y familias, dando cuenta de la complejidad que las organiza y de la existencia de diversos posicionamientos entre los sujetos docentes. En el caso de instituciones que trabajan con alumnos en situación de pobreza, algunas investigaciones de corte cualitativo-interpretativo han mostrado que los profesores y maestros apuntan que los padres tienen "poco interés" por la escolarización de sus hijos, a la vez que ponen de manifiesto las dificultades para lograr que los padres se acerquen y establezcan algún vínculo con la institución (TIRAMONTI y MINTEGUIAGA, 2004; ACHILLI, 2010). Asimismo, y según estos trabajos, los docentes y directivos conciben una cierta "debilidad disciplinadora" de las familias, la que deviene en una socialización "ineficaz" para la incorporación de sus hijos a los patrones de conducta socialmente aceptados. En el marco de estas coordenadas, el trabajo de "contención" que realiza la escuela se vincula a una intención de control social y adquiere ribetes afectivos y relativos a suplir una supuesta "falta de afecto" por parte del entorno social y familiar de los alumnos (Redondo, 2004). En algunas investigaciones se ha dado cuenta de que el afecto parece tener una circulación contradictoria en las escuelas, como un componente de la creciente "afectivización" de las relaciones pedagógicas (ABRAMOWSKI, 2010), pero también como algo que se da o se quita frente a algunos grupos o conductas. En este marco, las relaciones entre escuelas y familias han sido caracterizadas como oscilantes y paradójicas, dando cuenta de posicionamientos que pueden resultar contradictorios en los mismos sujetos (SOUTHWELL, 2008). 
Desde el campo de la antropología social, la investigación etnográfica de Cerletti (2010) muestra que familias que viven en condiciones de pobreza otorgan un lugar central a la educación de los niños como posibilidad de ascenso social. Muestra una amplia heterogeneidad de sentidos que las familias atribuyen a la escolarización de sus hijos en sus prácticas de elección educativa, dando cuenta de procesos de búsqueda activa por parte de las familias de una educación escolar vista como fundamental para el desarrollo de las experiencias formativas de los niños. En consonancia con estos planteos, la investigación de Redondo (2004) muestra que, para los sectores populares, la escuela sigue siendo una oportunidad y continúa siendo cargada de expectativas, aún en tiempos de ausencia de promesas de ascenso social. En una línea similar, en su análisis de los vínculos entre escuela y familias en contextos de desigualdad social, Achilli (2010) plantea la hipótesis de que ambos ámbitos se construyen en procesos dialécticos y de mutuas penetraciones y condicionamientos, que estas características relacionales son constitutivas de las identidades sociales, tanto de los maestros como de los aprendices escolarizados.

\section{ENTRE LA ADECUACIÓN Y LA DISPUTA AL CONTEXTO: DEBATES EN TORNO DE LOS VÍNCULOS ENTRE EL TRABAJO DOCENTE Y LAS DESIGUALDADES SOCIALES}

Otra línea de debates que ha atravesado las producciones académicas recientes en el campo de la investigación acerca de los vínculos entre trabajo docente, escolarización y desigualdad en Argentina se ha referido a la conveniencia, pertinencia, viabilidad y derecho por parte de los niños/as a recibir una "formación situada", que atienda "al contexto" en el que viven. La "adecuación al contexto" y las oposiciones entre éste y la lógica escolar han sido destacadas por algunas investigaciones que señalan que se tiende a expresar un juicio negativo y pesimista sobre los alumnos y su interés y capacidad para aprender, fundando estas asunciones en problemas de disciplina, motivación y acompañamiento por parte de las familias de los estudiantes, en el marco del supuesto antagonismo entre lo popular y lo escolar (TENTI FANFANI, 2006). Estas situaciones entran en tensión con el trabajo de enseñanza y son el disparador de diferentes resoluciones, entre las que se cuenta la adaptación de los contenidos curriculares a las características y posibilidades de los niños sin reducir las exigencias de aprendizaje, constituyendo ello un dilema para los docentes. En un estudio reciente (ALLIAUD y 
ANTELO, 2009) se analiza el modo en que se construye, por parte de los docentes, un conjunto de problemas en torno de la idea de dar más al que menos tiene -en términos de subsistencia, contención familiar y "reglas" y "normas". A partir de estos diagnósticos, suelen desarrollarse iniciativas tales como plantear la enseñanza en función del contexto, adecuar el currículum a las necesidades de los alumnos y recurrir al apoyo de especialistas.

Esas propuestas se han fundado en la idea de que las diferencias resultan desigualdades y deben recibir una atención específica. Algunas investigaciones recientes de corte interpretativo han debatido con estas premisas, mostrando que la experiencia del contexto como limitante de toda posibilidad educativa no invade de un modo absoluto los cotidianos escolares (ACHILLI, 2010; REDONDO, 2004; REDONDO y THISTED, 1999; SOUTHWELL, 2008; VASSILIADES, 2012). Ellas dan cuenta de prácticas escolares por parte de los y las docentes que prefiguran tensiones y puestas en marcha de desafíos y utopías que sostienen la construcción de otros horizontes para los/as estudiantes que asisten a las escuelas que trabajan con sujetos que viven en condiciones de pobreza.

Como parte de sus contribuciones, algunos estudios han afirmado la necesidad de desarrollar un enfoque "situacional" y diversas metodologías de formación de profesores para situaciones "potencialmente conflictivas", planteando la necesidad de que haya una preparación específica para el trabajo con sectores populares (VELEDA, RIVAS y MEZZADRA, 2011). Esta posición ha sido discutida por varios trabajos, algunos de los cuales analizan un conjunto de propuestas de formación de grado y posgrado de principios de la década pasadadirigidas a los docentes (SERRA y CANCIANO, 2002). Se trata de estrategias formalizadas que se proponen preparar a los profesores para educar a los pobres, convirtiendo a la pobreza en un rasgo diferencial del otro, una situación del orden de lo particular que reorganiza los elementos de la relación pedagógica (SERRA, 2002). El supuesto sobre el que se sostienen estas propuestas es que es necesaria una formación específica para trabajar con la pobreza, en tanto la formación docente resultaría insuficiente para ello. Una segunda asunción que estructura estas iniciativas es que existiría un "déficit", relativo al entorno social de los niños y sus familias, que requiere una preparación especial por parte de los docentes.

De acuerdo con Serra (2002), estas operaciones invierten el modo en que se ha construido históricamente la relación entre educación y pobreza, en el que la escuela apostaba a la inclusión y la 
enseñanza del conjunto de la población, realizando una operación con aquello que era considerado del orden de la desigualdad. Esta premisa es desplazada por cursos y propuestas formativas estructuradas en torno de la problemática de la pobreza abordada en términos de "riesgo social", convalidando la ruptura de la aspiración a instituir algo del orden de lo común y renunciando a éste como expresión de la construcción de un universal (SERRA, 2003).

En esta línea, una serie de producciones académicas recientes han profundizado las críticas a las propuestas de adecuación de la formación y el trabajo docente a las situaciones "de pobreza". Desde una óptica que privilegia problemas específicos del campo de la pedagogía para la indagación sobre la tarea de enseñar-como la transmisión cultural o la construcción de vínculos intergeneracionalesuna serie de trabajos ha analizado los modos en que la cuestión de la igualdad depende de lo que los maestros hagan con sus estudiantes en la escuela, de cómo los reciban y los alojen considerándolos iguales y con iguales derechos a ser educados y aprender (DUSSEL y SOUTHWELL, 2004). En efecto, si bien las instituciones escolares pueden desempeñar un papel en la construcción de desigualdad y la injusticia en nuestras sociedades, también resulta necesario recuperar una pregunta por lo político en el trabajo de enseñar y por la construcción de lo común y lo colectivo (BIRGIN, 2006, 2014; SOUTHWELL, 2009) en pos de habilitar otros horizontes para quienes participan de los procesos de escolarización. Así, estos estudios habilitan la posibilidad de sumar un interrogante más a los problemas de investigación mencionados: cómo los procesos de escolarización y el trabajo docente pueden involucrarse en la construcción cotidiana de la igualdad.

\section{CONCLUSIONES}

A lo largo de este artículo he procurado realizar un análisis crítico y sistemático de la producción científica acerca de los vínculos entre trabajo docente, escolarización y desigualdad en el período 1985-2015 con el objeto de examinar los principales debates conceptuales y teóricometodológicos en la investigación, como así también sus principales contribuciones para las discusiones actuales en el campo de la educación.

El recorrido por los principales núcleos de discusión ha mostrado cómo la reformulación de los abordajes estructurales ha permitido redefinir los modos en que se conceptualizan los vínculos entre la escolarización, el trabajo docente y la desigualdad, abriendo 
la posibilidad de considerar el punto de vista de los sujetos y las instituciones educativas para abordar estas construcciones desde otras coordenadas teórico-metodológicas, ligadas a la comprensión interpretativa de la cotidianeidad escolar.

Asimismo, las discusiones surgidas de la incorporación de perspectivas centradas en la heterogeneidad y el dinamismo de las identidades permitió abordar el carácter diverso de los procesos y dinámicas de escolarización, como así también la multiplicidad de sentidos en torno del trabajo docente y las desigualdades sociales. Estos movimientos habilitaron la posibilidad de mostrar un amplio abanico de posiciones de sujeto de los maestros, profesores, directores y familias, permitiendo la construcción de nuevos problemas de investigación en torno de estas temáticas. Entre ellos, cabe destacar las perspectivas que abrevan en la idea de que las nociones de igualdad y desigualdad suponen una relación, descartando la posibilidad de considerarlas como esencias fijas e inmutables. Al no ser un estado fijado de antemano, la igualdad implica un conjunto de vínculos de los que es necesario dar cuenta.

Finalmente, en los debates respecto del lugar de los contextos de pobreza en las instancias de formación y trabajo docente, el artículo ha mostrado el modo en que se han desarrollado un conjunto de producciones académicas y científicas que aportaron nuevos modos de aproximarse a los vínculos entre docencia, escolarización y desigualdad. Estas perspectivas incorporan preguntas propias del campo de la pedagogía, como las relativas a la transmisión cultural o a los vínculos intergeneracionales, que renuevan los debates anteriores y abren interrogantes en relación a los modos en que el trabajo docente y los procesos de escolarización pueden vincularse con la construcción de horizontes más igualitarios.

\section{BIBLIOGRAFÍA}

ABRAMOWSKI, A. Maneras de querer. Los afectos docentes en las relaciones pedagógicas. Buenos Aires: Paidós, 2010.

ACHILLI, E. Escuela, familia y desigualdad social. Una antropología en tiempos neoliberales. Rosario: Laborde Edito, 2010.

ALLIAUD, A. y ANTELO, E. Los gajes del oficio. Enseñanza, pedagogía y formación. Buenos Aires: Aique, 2009.

Arroyo, M. ¿Hay en la escuela algo que tenga que ver con un proyecto común? En: TIRAMONTI, G. (comp.), La trama de la desigualdad educativa. Mutaciones recientes en la escuela media (p.119-146).Buenos Aires: Manantial, 2004.

BAUDELOT, C. y ESTABLET, R. La escuela capitalista en Francia. Madrid: Siglo XXI, 1976. 
BIRGIN, A. Pensar la formación de los docentes en nuestro tiempo. En: Terigi, F. (comp.), Diez miradas sobre la escuela primaria (p. 267-294). Buenos Aires: Fundación OSDE Siglo XXI Editores, 2006.

BIRGIN, A. (coord.). Estudio sobre criterios de calidad y mejora de la formación docente del MERCOSUR. Buenos Aires: Teseo, 2014.

BOWLES, S. y GINTIS, H. La instrucción escolar en la América capitalista. México: Siglo XXI, 1976.

BRASLAVSKY, C. La discriminación educativa en la Argentina. Buenos Aires: FLACSO - Miño y Dávila, 1985.

CERLETTI, L. Una etnografía sobre las relaciones entre familias y las escuelas en contextos de desigualdad social (Tesis Doctoral). Facultad de Filosofía y Letras, Universidad de Buenos Aires, Buenos Aires, 2010.

DUSSEL, I. y SOUTHWELL, M. La escuela y la igualdad: renovar la apuesta. En: E1 Monitor de la Educación, 5(1). Buenos Aires: Ministerio de Educación, Ciencia y Tecnología de la Nación, p. 1-4, 2004.

DUSSEL, I. Desigualdades sociales y desigualdades escolares en la Argentina de hoy. Algunas reflexiones y propuestas. En: TEDESCO, J. C. (comp.), ¿Cómo superar la desigualdad y la fragmentación del sistema educativo argentino? (p. 85-116). Buenos Aires: IIPE UNESCO, 2005.

DUSSEL, I. Impactos de los cambios en el contexto social y organizacional del oficio docente. En: TENTI FANFANI, E. (comp.), El oficio de docente. Vocación, trabajo y profesión en el siglo XXI (p.143-174). Buenos Aires: Siglo XXI, 2006.

HILLERT, F. A modo de conclusión. En: HILLERT, F., AMEIJEIRAS, M. J. y GRAZIANO, N. (comp.), La mirada pedagógica para el siglo XXI: teorías, temas y prácticas en cuestión. Reflexiones de un encuentro (p. 205-210). Buenos Aires: Editorial de la Facultad de Filosofía y Letras de la Universidad de Buenos Aires, 2009.

KAPLAN, C. Buenos y malos alumnos: descripciones que predicen. Buenos Aires: Aique, 1992. KAPLAN, C. La inteligencia escolarizada. Buenos Aires: Miño y Dávila, 1997.

KAPLAN, C. Talentos, dones e inteligencias. El fracaso escolar no es un destino. Buenos Aires: Colihue, 2008.

LLOMOVATTE, S. A modo de presentación. En: HILLERT, F., AMEIJEIRAS, M. J. y GRAZIANO, N. (comp.), La mirada pedagógica para el siglo XXI: teorías, temas y prácticas en cuestión. Reflexiones de un encuentro (pp. 9-11). Buenos Aires: Editorial de la Facultad de Filosofía y Letras de la Universidad de Buenos Aires, 2009.

MORGADE, G. El determinante de género en el trabajo docente de la escuela primaria. Cuadernos IICE Nro. 12. Buenos Aires: Miño y Dávila Editores, 1992.

MORGADE, G. Aprender a ser mujer, aprender a ser Varón. Relaciones de género y educación. Esbozo de un programa de acción. Buenos Aires: Ediciones Novedades Educativas, 2001.

MORGADE, G. Sexualidad y prevención: discursos sexistas y heteronormativos en la escuela media. En: Revista del Instituto de Ciencias de la Educación, 24. Buenos Aires: Facultad de Filosofía y Letras de la Universidad de Buenos Aires, p. 27-3, 3, 2006. 
PADAWER, A. Cuando los grados hablan de desigualdad. Buenos Aires: Teseo, 2008.

POLIAK, N. Reconfiguraciones recientes en la educación media: escuelas y profesores en una geografía fragmentada. En: Tiramonti, G. (comp.), La trama de la desigualdad educativa. Mutaciones recientes en la escuela media (p. 147-192). Buenos Aires: Manantial, 2004.

REDONDO, P. Escuelas y pobreza. Entre el desasosiego y la obstinación. Buenos Aires: Paidós, 2004.

REDONDO, P. y THISTED, S. Las escuelas 'en los márgenes'. Realidades y futuros. En: PUIGGRÓS, A. (comp.), En los límites de la educación. Niños y jóvenes del fin de siglo. Buenos Aires: Homo Sapiens, 1999.

SERRA, S. En el nombre del pobre. En: AA.VV. Lo que queda de la escuela (p. 101-109). Rosario: Laborde Editor, 2002.

SERRA, S. y CANCIANO, E. Formación docente y riesgo social: la pobreza en el/ del discurso pedagógico. Trabajo presentado en el Ateneo Universitario "Derechos Humanos, Pobreza y Exclusión”. Universidad Nacional de Rosario, Rosario, 2002.

SOUTHWELL, M. La tensión desigualdad y escuela. Breve recorrido de sus avatares en el Río de la Plata. En: MARTINIS, P. y REDONDO, P. (comp.), Igualdad y educación. Escrituras entre (dos) orillas (p. 103-122). Buenos Aires: Del Estante Editorial, 2006.

SOUTHWELL, M. Hacer escuela con palabras: directores de escuela media frente a la desigualdad. En: Revista Archivos de Ciencias de la Educación 2(2). La Plata: FaHCEUNLP, 2008.

SOUTHWELL, M. Docencia, tradiciones y nuevos desafíos en el escenario contemporáneo. En: Yuni, J. (comp.), La formación docente. Complejidad y ausencias (p. 169-199). Córdoba: Encuentro Grupo Editor, 2009.

SOUTHWELL, M. y VASSILIADES, A. El concepto de posición docente: notas conceptuales y metodológicas. En: Revista Educación, Lenguaje y Sociedad, 11. General Pico: Instituto para el Estudio de la Educación, el Lenguaje y la Sociedad, p. 163-187, 2014.

SUÁREZ, D. La tradición crítica en educación y reconstrucción de la pedagogía”. En: ELISALDE, R. y AMPUDIA, M. (comp.) Movimientos sociales y educación: teoría e historia de la educación popular en Argentina y América Latina (p. 37-52). Buenos Aires: Buenos Libros, 2008.

TENTI FANFANI, E. La escuela vacía. Buenos Aires: UNICEF - Losada, 1993.

TENTI FANFANI, E. Profesionalización docente: consideraciones sociológicas. En: TENTI FANFANI, E. (comp.), El oficio de docente. Vocación, trabajo y profesión en el siglo XXI (p. 119.142). Buenos Aires: Siglo XXI, 2006.

TIRAMONTI, G. La fragmentación educativa y los cambios en los factores de estratificación. En: TIRAMONTI, G. (comp.), La trama de la desigualdad educativa. Mutaciones recientes en la escuela media (p.15-46). Buenos Aires: Manantial, 2004.

TIRAMONTI, G. y MINTEGUIAGA, A. Una nueva cartografía de sentidos para la escuela. En: TIRAMONTI, G. (comp.), La trama de la desigualdad educativa. Mutaciones recientes en la escuela media (p.101-118). Buenos Aires: Manantial, 2004.

VASSILIADES, A. Regulaciones del trabajo de enseñar en la provincia de Buenos Aires: posiciones docentes frente a la desigualdad social y educativa. (Tesis de Doctorado) Buenos Aires: Facultad de Filosofía y Letras de la Universidad de Buenos Aires, 2012. 
VELEDA, C., RIVAS, A. y MEZZADRA, F. La construcción de la justicia educativa. Criterios de redistribución y reconocimiento para la educación argentina. Buenos Aires: CIPPEC-UNICEF-Embajada de Finlandia, 2011.

\section{NOTAS}

${ }^{1}$ Este artículo presenta resultados de la realización de mi tesis de doctorado "Regulaciones del trabajo de enseñar en la provincia de Buenos Aires: posiciones docentes frente a la desigualdad social y educativa”, dirigida por la Dra. Myriam Southwell y desarrollada en la Facultad de Filosofía y Letras de la Universidad de Buenos Aires, y del trabajo en curso en el marco del proyecto "El trabajo de enseñar en tiempos de la Asignación Universal por Hijo: posiciones docentes frente a las desigualdades sociales y educativas", que actualmente llevo adelante en el marco de la Carrera de Investigador Científico y Tecnológico del Consejo Nacional de Investigaciones Científicas y Técnicas (CONICET) de Argentina.

${ }^{2}$ En relación a esta cuestión, este artículo recupera intercambios y discusiones compartidas en los ámbitos del Proyecto UBACyT 2014-2017 “La conformación del campo pedagógico: sentidos y disputas en torno de las desigualdades y diferencias en educación” y del Seminario de Investigación Educativa "Desigualdades y diferencias en el campo pedagógico" -cuyo dictado compartimos con Sofía Thisted, Lucía Caride y María Inés Maañon-, ambos en la Facultad de Filosofía y Letras de la Universidad de Buenos Aires.

Presentado: 03/12/2016

Aprobado: 31/07/2017

Contacto:

Instituto de Investigaciones en Humanidades y Ciencias Sociales Calle 51 entre 124 y 125. Edificio C. Oficina 208 a 211. (1925) Ensenada. Provincia de Buenos Aires. Argentina. 